\title{
Major orbital complications of endoscopic sinus surgery
}

\author{
C Rene, G E Rose, R Lenthall, I Moseley
}

\begin{abstract}
Background-The paranasal sinuses are intimately related to the orbit and consequently sinus disease or surgery may cause severe orbital complications. Complications are rare but can result in serious morbidity, the most devastating of which is severe visual loss.

Methods-A retrospective review was undertaken of four cases of severe orbital trauma during endoscopic sinus surgery. Results-All the cases suffered medial rectus damage, one had additional injury to the inferior rectus and oblique, and two patients were blinded as a result of direct damage to the optic nerve or its blood supply.

Conclusion-Some ophthalmic complications of endoscopic sinus surgery are highlighted, the mechanisms responsible are discussed, and recommendations for prevention, early recognition, and management are proposed.
\end{abstract}

(Br f Ophthalmol 2001;85:598-603)

Sinus surgery aimed at enhancing sinus drainage and aeration and at the extirpation of diseased mucosa has long been used for managing a wide variety of conditions. The intimate relation between the paranasal sinuses and the orbit places the orbital contents at risk of injury during sinus surgery, especially surgery of the ethmoid sinuses. ${ }^{1-6}$ The introduction of transnasal endoscopic sinus surgery in recent years presents a greater risk for orbital injury, especially during ethmoidal surgery with a less experienced surgeon.

\section{Case reports}

CASE 1

A 52 year old man underwent a right transnasal ethmoidectomy for polyposis and awoke with diplopia, the right eye being divergent with a total absence of adduction and a large subconjunctival haemorrhage was present without proptosis.

The patient was referred to the orbital clinic 11 days after surgery with persistent diplopia and a slight change in colour perception by the right eye. His acuities were Snellen 6/4 in each eye, Ishihara colour discrimination was normal, and there was no relative afferent pupillary defect. The right eye was markedly exotropic and was unable to reach the midline with maximal adduction (Fig 1A-C), although all recti appeared to have adequate perfusion
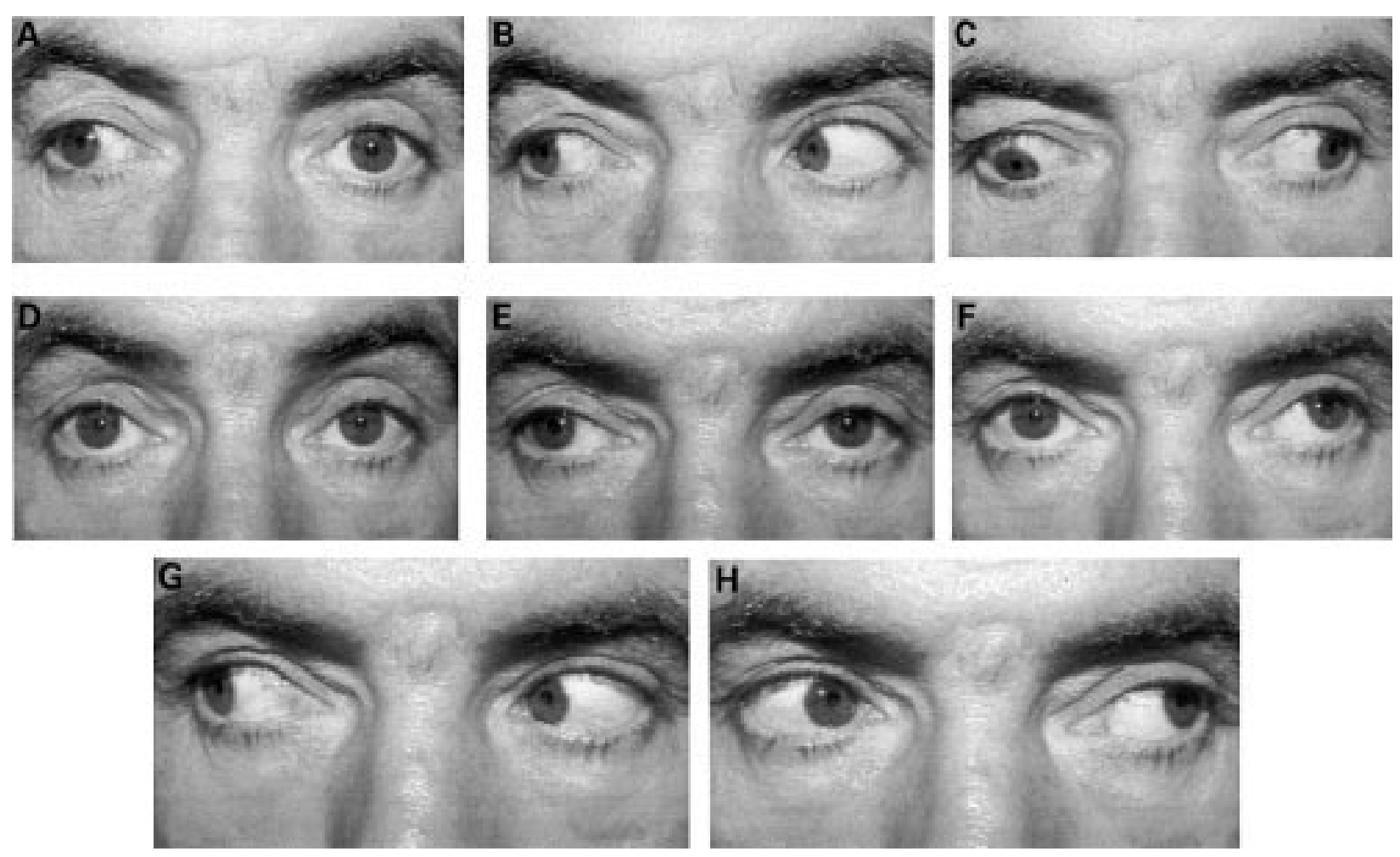

Figure 1 Case 1: a 52 year old man who presented 11 days after right orbital entry during endonasal ethmoidectomy showing (A) marked right exotropia and (B) good right abduction but no adduction of the right eye with (C) marked hypotropia on attempted left gaze. Nine months after orbital repair he had intermittent exotropia $(D-F)$ and markedly improved right ocular ductions $(G, H)$. 

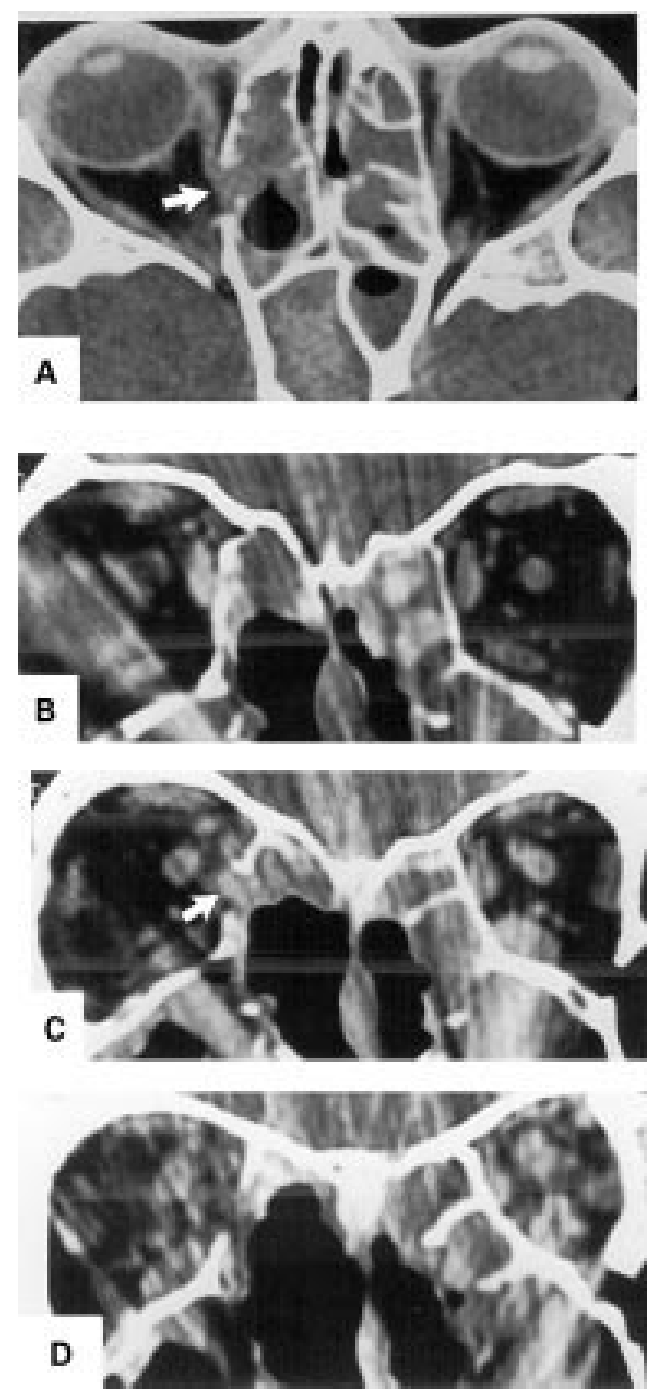

Figure 2 Case 1: (A) axial CT scan showing a defect in the right lamina papyracea to which the medial rectus appears adherent (arrow); the eye is abducted and there is minor enophthalmos. Only the anterior part of the muscle can be seen but this could be because of the plane of image. There is extensive sinus disease. (B)-(D) Direct coronal sections showing a large air containing postoperative space in continuity with the right nasal cavity. The medial rectus muscle is clearly visible in (B) just in front of the defect; its high density is artefactual. The muscle is seen to be displaced medially (arrow) into the defect in $(C)$ and cannot be discerned in (D) which passes through its posterior end.

with no stagnation of blood flow in episcleral arterioles. There was no proptosis or enophthalmos, and cutaneous sensation, pupillary reaction, and fundal examination were all normal.

On computed tomographic (CT) scanning (Fig 2) and magnetic resonance imaging (MRI) a defect was seen in the medial wall of the right orbit, adjacent to an air filled cavity in the position of the partially removed ethmoid sinuses, the septa of which were also defective. The medial rectus was deviated medially and was adherent to this defect; the muscle was not visible posterior to this area of adhesion.

A right transcutaneous medial orbitotomy was performed under general anaesthesia at which time passive abduction of the globe was found to be limited. Orbital fat was found adherent to the breach in the lamina papyracea with incarceration of the medial rectus; the tissues were freed from the site of injury and the defective wall repaired with silicone sheet. Immediately after surgery the right eye remained exotropic with poor adduction, but adduction improved over 9 months so that the patient could fuse in the primary position for most of the time (Fig 1D-H, Fig 3). Persistent intermittent exotropia (Fig 1D-F) 16 months after orbital repair was treated with left lateral rectus recession.

CASE 2

After bilateral intranasal endoscopic sinus surgery, a 46 year old man awoke with gross right periorbital swelling and bruising and a temporary tarsorrhaphy. Diplopia, much worse in the primary position and on left gaze, had persisted since the surgery.

Fourteen months later he presented to the orbital service at which time his unaided acuities were Snellen 6/4 in both eyes with no optic neuropathy. A left head turn was adopted to overcome a significant right divergent strabismus and to use a small field of single vision on far right gaze; with attempted adduction the right eye could not cross the midline (Fig $4 \mathrm{~A}-\mathrm{C})$. The forced duction test was negative and a force generation test revealed almost no medial rectus function. Two millimetres of right relative enophthalmos were present but the rest of the ocular and periorbital examination was normal.

An axial CT scan showed enophthalmos and abduction of the right globe with prolapse of orbital fat and soft tissue into a large defect in the lamina papyracea (Fig 5). The bony defect extended to about $1 \mathrm{~cm}$ from the orbital apex; in addition, the medial wall of the maxillary antrum had been removed with only the inferior turbinate remaining. There were marked radiological abnormalities of the ethmoid and sphenoid sinus mucosa. On both axial and coronal images the right medial rectus could not be identified at the level of, or anterior to, the bony defect, suggesting that it had been divided. More posteriorly it appeared thickened, as if bunched up, and displaced superiorly with overriding of the superior oblique muscle.

At transconjunctival orbitotomy the medial rectus was found to be adherent to the site of orbital injury, with about two thirds of the upper border of the muscle being absent (Fig 4D). The incarcerated muscle remnant was dissected free and right medial rectus resection was combined with lateral rectus recession, there being an improved position and size of the field of single vision after surgery.

\section{CASE 3}

During endoscopic surgery for chronic nasal polyposis the left orbit of a 50 year old man was inadvertently entered, resulting in loss of left vision and a gross left exotropia.

One year later he was examined in the orbital service when his right acuity was Snellen 6/4 and the left was blind with a dense left relative afferent pupillary defect. There was deepening 

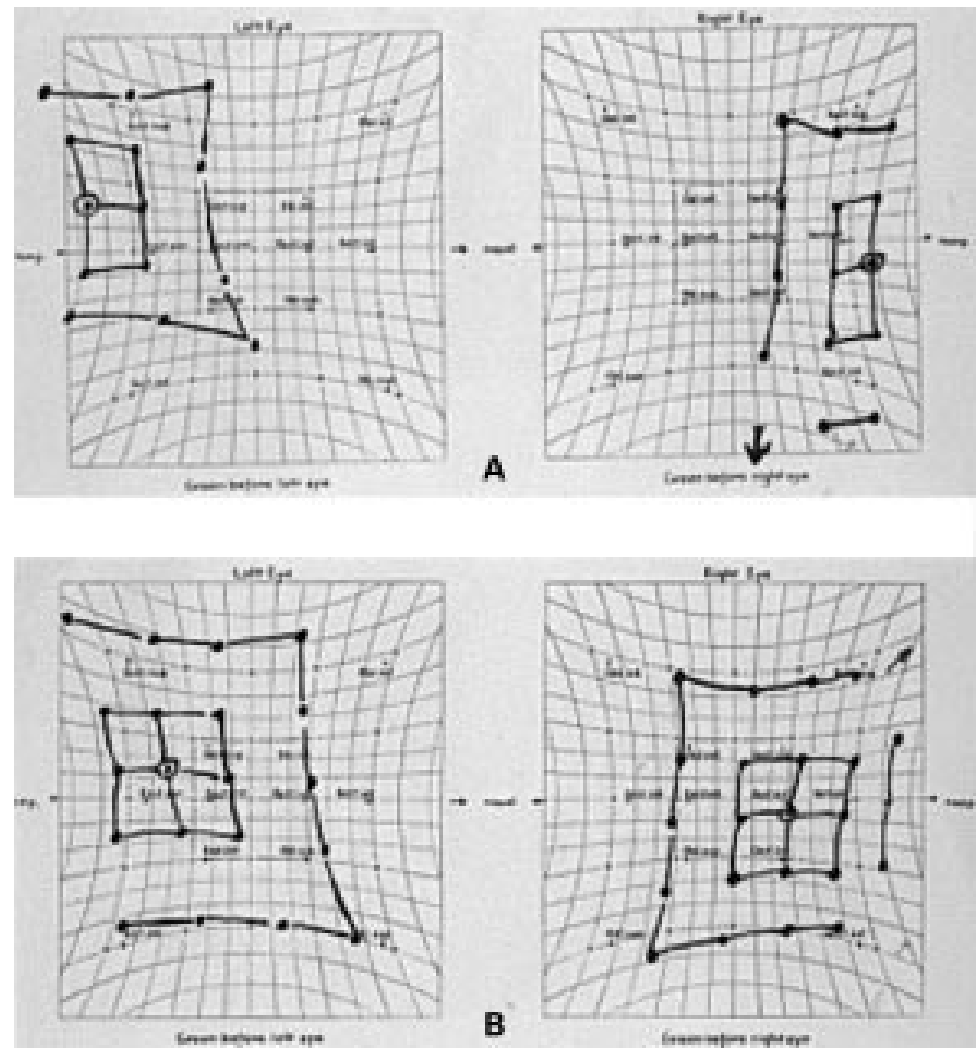

Figure 3 Case 1: Hess charts (A) 6 weeks and (B) 8 months after orbital surgery showing delayed recovery of medial rectus function after release of the entrapped muscle.

of the left upper lid sulcus and $3 \mathrm{~mm} \mathrm{left}$ enophthalmos with marked left divergence and almost no horizontal ductions (Fig 6). There was total optic atrophy and retinal arteriolar sheathing on the affected side.

Axial CT scanning showed left enophthalmos with divergent gaze. The optic nerve was sharply angulated medially just behind the globe and, behind this, it was closely applied to the medial wall of the orbit in the normal position of the medial rectus (Fig 7). The middle portion of the lamina papyracea was defective and one or two small specks of bone density were seen in the vicinity of the optic nerve. The medial rectus muscle could not be identified at the level of the bone defect and appeared to be distorted both anterior and posterior to it. The corresponding coronal image confirmed the adherence of the optic nerve to the bone defect and indicated bilateral ethmoid, maxillary sinus and intranasal inflammatory disease.

CASE 4

A 55 year old man underwent endoscopic sinus surgery for recurrent postnasal drip and nasal obstruction. A breach of the lamina papyracea with prolapse of orbital fat was suspected during right sided surgery and the ipsilateral pupil was noted to be dilated, but reacted to light. There was no perception of light in the right eye immediately after surgery and the pupillary response to direct illumination was poor. After ophthalmic consultation, treatment with systemic acetazolamide, mannitol, dexamethasone, nifedipine, and xanthine was instituted on the presumption of ophthalmic arterial spasm. There is no record of the ocular
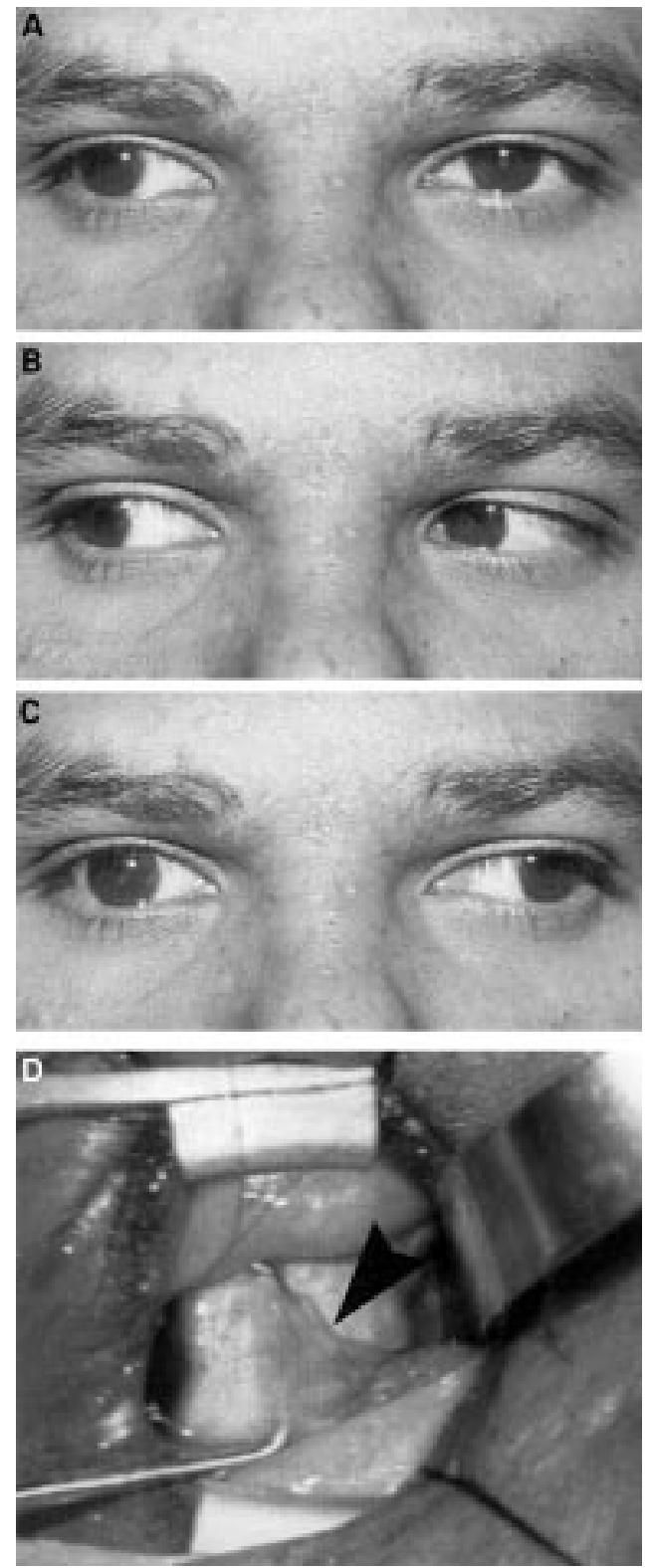

Figure 4 Case 2: presentation at 14 months after right orbital injury during endoscopic sinus surgery with $(A)$ right exotropia, (B) normal right abduction, and (C) an inability to reach the midline on attempted adduction of the right eye. (D) View of the anterior part of the right medial rectus showing loss of most muscle fibres (arrow) in the anterior third of the muscle at the site of previous orbital injury.

position or ductions. After MRI scanning on the first postoperative day, further lamina papyracea was removed in an attempt to decompress the right orbit.

The patient was referred to the orbital clinic on the fourth day after the initial surgery. The right eye was blind with a dense relative afferent pupillary defect and in gross divergence with no effective adduction. There was widespread subconjunctival haemorrhage (Fig $8 \mathrm{~A}$ ) and the right fundus revealed severe retinal oedema and a "cherry red spot" macula with no retinal arterial perfusion (Fig 8B).

On axial T1 and T2 weighted MRI images there was about $4 \mathrm{~mm}$ of right enophthalmos with divergent gaze. The right medial rectus could not be identified and the medially 

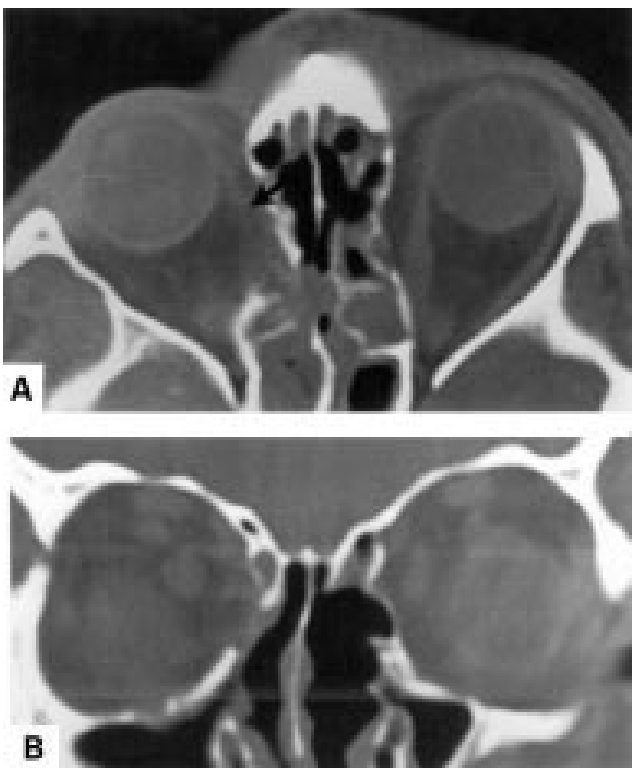

Figure 5 Case 2: $(A)$ axial and $(B)$ direct coronal $C T$ scans showing marked enophthalmos and abduction of the right eye with prolapse of orbital soft tissue into a defect in the lamina papyracea. There has been extensive nasal surgery. The medial rectus cannot be identified at the level of the bone defect but, more anteriorly, small threads of tissue are seen in its normal position ( $A$, arrow).

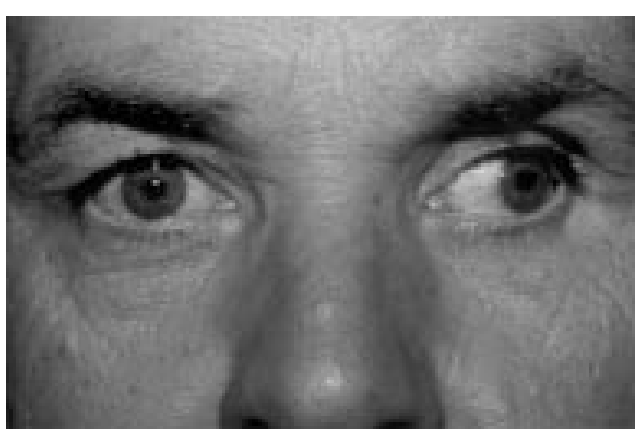

Figure 6 Case 3: orbital entry during endoscopic sinus surgery a year before presentation has resulted in a

markedly sunken and blind left eye with a divergent squint and no adduction.

deviated right optic nerve was seen with difficulty. A fluid level was noted in the sphenoid sinus. On the coronal sections the right medial rectus could not be identified on any image. There was prolapse of the anterior portion of the right optic nerve towards a defect in the inferomedial wall of the orbit (Fig 9). Posteriorly, the normal cerebrospinal fluid surrounding the optic nerve could not be seen and there was diffuse high signal in the inferior part of the orbit, suggesting transection of the sheath with egress of fluid into the orbital fat. Diffuse inflammatory changes were present in the nasal cavity and maxillary sinuses.

\section{Discussion}

The proximity of the paranasal sinuses to the orbits exposes the orbital contents to the risk of inadvertent trauma during sinus surgery. The orbit is at greatest risk during ethmoid sinus surgery ${ }^{1-6}$ because the lamina papyracea, especially at the extremes of age, is extremely thin and may be incomplete in some patients; such a thin wall presents an almost imperceptible
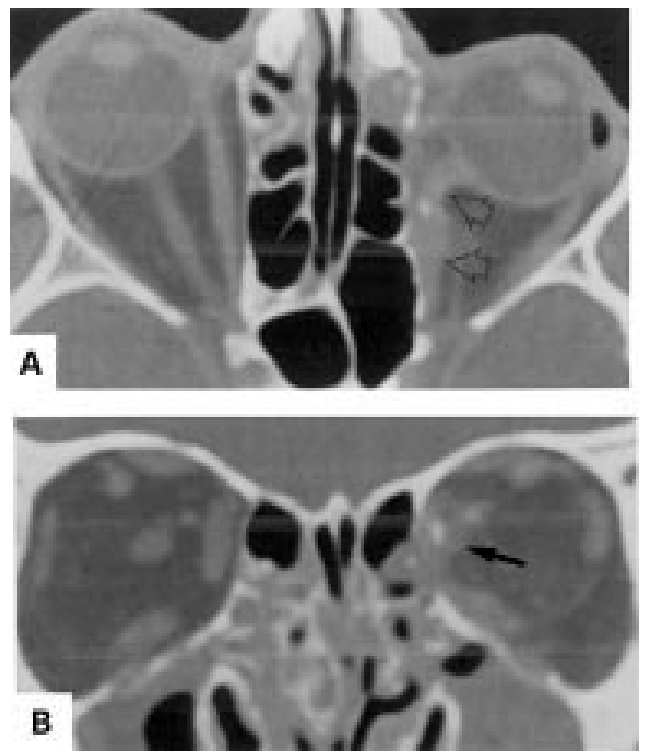

Figure 7 Case 3: (A) axial CT scan showing the left optic nerve (open arrows) in abnormal position, angulated just behind the eye and inseparable from soft tissue along the lamina papyracea. A small dense area presumably represents a flake of bone driven into the orbit. There is $5 \mathrm{~mm}$ left enophthalmos and the eye is abducted. The direct coronal scan (B) shows the optic nerve (arrow) and the bone fragment to form part of a mass of soft tissue density which, through a defect in the lamina papyracea, is continuous with tissue in the ethmoid sinuses. There is widespread sinus and nasal disease.
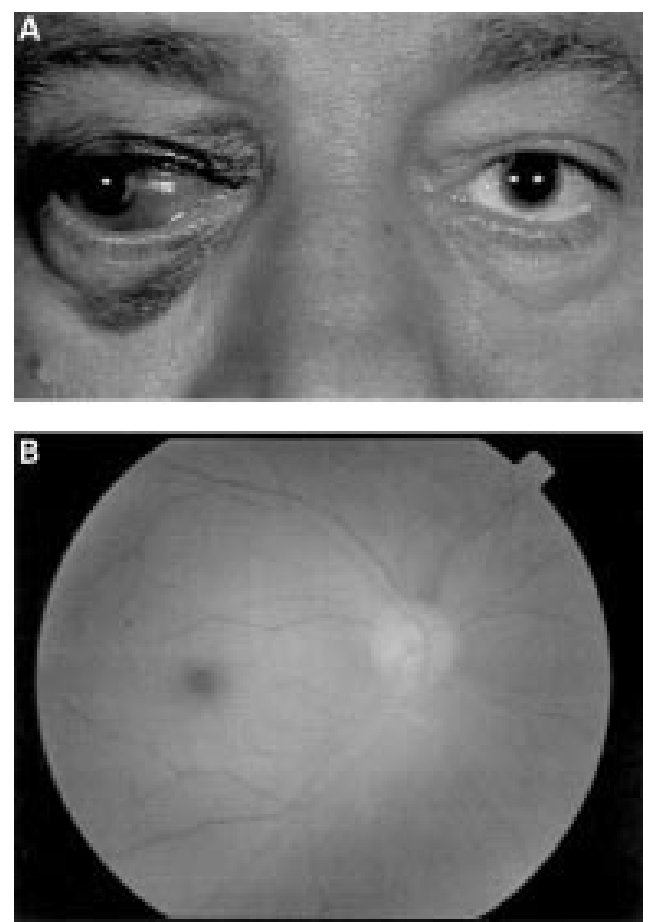

Figure 8 Case 4: $(A)$ a 55 year old man presenting with a divergent blind right eye 4 days after sinus surgery; there is an extensive subconjunctival haemorrhage and eyelid bruising. Red-free photograph of the right fundus (B) shows the foveal sparing retinal oedema of an acute central retinal artery occlusion; poor image quality is the result of anterior chamber cellular reaction.

boundary during sinus surgery. Although the sinus instrumentation used during surgery in the described cases is unknown, the lamina papyracea is especially at risk when approached from the nasal space (through the 

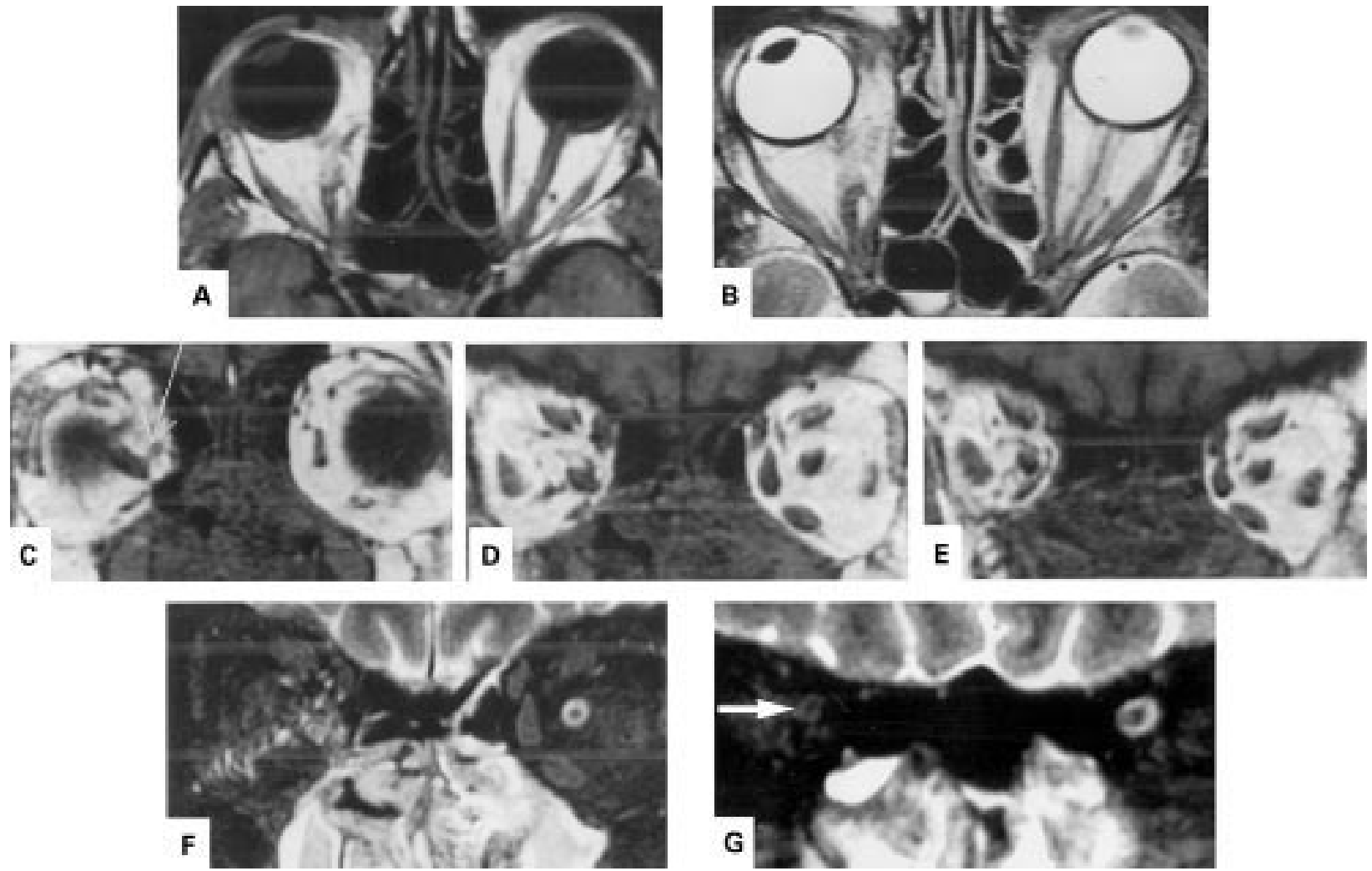

Figure 9 Case 4: $(A)$ and (B) axial T1 and T2 weighted images showing $4 \mathrm{~mm}$ right enophthalmos and abduction. The right medial rectus muscle cannot be identified and the anterior portion of the medially deviated optic nerve is difficult to see. There is a fluid level in the right side of the sphenoid sinus in (B). (C)-(E) Coronal T1 weighted images (anterior to posterior) show a structure just behind the eye ((C), electronic arrow) which can be traced back to the optic nerve/sheath complex in (E), intimately related to a bone defect; there is no clear demonstration of the medial rectus muscle. The paranasal sinus and nasal cavity return low signal compatible with swollen mucosa. The coronal T2 weighted image through the mid orbit (F) shows extensive high signal extending from the area of the bone defect across the lower part of the retrobulbar fat and, more posteriorly (G), the optic nerve sheath (arrow) appears collapsed, suggesting transection of the sheath and leakage of cerebrospinal fluid into the fat.

diseased sinus) rather than from the extraperiorbital space, a plane that is clearly defined during external ethmoidectomy. The risk of breach is probably also greater when there are anatomical variants in the sinuses ${ }^{7}$ or when mechanised systems are used for sinus debridement. Mechanised systems extract tissue very rapidly and provide no tactile feedback to the surgeon about the type of tissue being removed. None of these patients had prior sinus surgery, although disruption of normal anatomy resulting from disease or previous surgery might also predispose to inadvertent orbital entry. ${ }^{5}$

The medial rectus lies close to the lamina papyracea and was injured in all patients in this series with direct loss of muscle (cases 2-4; Figs 4D, 5, 7 and 9) or with entrapment (case 1; Fig 2). The injury is associated with the very characteristic gross divergent strabismus (Figs 1A, 6 and 8A) and almost absent adduction (Figs 1C and 4C) within the injured orbit. It is also likely that damage to the nerve or vascular supply to the muscle may affect its function, the former mechanism being compatible with the slow (9 month) recovery of adduction in case 1.

Two of the patients suffered unilateral blindness because of direct optic nerve damage (cases 3 and 4), a complication that has been reported only once before as bilateral blindness after endoscopic ethmoidectomy ${ }^{8}$ and once as a transient visual loss in the absence of direct optic nerve injury. ${ }^{9}$ Aerated posterior ethmoid air cells alongside the optic canal, so called Odoni cells, occur in a few people and these might place the optic nerve at greater risk of injury during ethmoidectomy. In the present series, however, the intraorbital optic nerve was damaged by instruments entering the orbit or by distraction of tissues from the orbit into the ethmoidectomy site (cases 3 and 4).

The incidence of ocular complications during sinus surgery is low but, as shown in this series, when they occur there may be significant morbidity. With recent interest in endoscopic sinus surgery, more ocular complications of such surgery are likely to present to the ophthalmologist. The risk of inadvertent orbital entry may be minimised by good preoperative assessment, including CT scanning to assess the extent of the sinus disease and to detect pre-existing anatomical variants. ${ }^{5710}$ Early intraoperative location of the lamina papyracea provides a key landmark, this being aided by measures to reduce operative bleeding, and modifications of surgical technique may significantly reduce the incidence of this complication. ${ }^{4}$

Intraorbital haemorrhage, either from direct damage to orbital vessels or by spread into the orbit through a medial wall defect, may cause an acute rise in orbital pressure with rapid onset of proptosis and loss of vision. ${ }^{911}$ Traction on the orbital fat may avulse an 
orbital vessel and cause intraorbital haemorrhage. For this reason it is prudent to keep the eyes uncovered during endoscopic sinus surgery and to monitor them for any movement resulting from traction on the orbital fat, this being an indication for immediate cessation of sinus surgery. Other indications of orbital entry include sudden swelling or bruising of the eyelids or the onset of proptosis. Severe intraorbital haemorrhage has also been thought to occur when a transected ethmoidal artery retracts into the orbit and continues to bleed. ${ }^{9}$

Direct optic nerve injury should be suspected if the pupil dilates rapidly during surgery (either from globe ischaemia or from damage to pupillomotor nerves) or if, after surgery, there is severe visual loss with a poorly reactive pupil and a relative afferent pupil defect. Severe orbital haemorrhage with secondary compressive optic neuropathy is characterised by rapidly increasing proptosis, subconjunctival haemorrhage, and periorbital ecchymosis; should it be recognised during surgery, a very tense orbit may require decompression by incision into the eyelid skin crease(s) and evacuation of haematoma from the orbital fat. Although frequently advocated, lateral canthotomy and inferior cantholysis may be insufficient to treat a major orbital haemorrhage.

A history of diplopia following sinus surgery and a motility defect is strongly suggestive of orbital entry during sinus surgery. Where there is clinically evident enophthalmos or muscle entrapment, strabismus surgery should be preceded by orbital exploration and release of entrapped tissues. If there is no medial rectus entrapment, consideration should be given to inducing paralysis of the lateral rectus with botulinum toxin to minimise contracture of the ipsilateral antagonist while awaiting recovery of the orbital soft tissue injury. Later repair of the large angle exotropia (or exophoria) may involve resection and/or advancement of the injured medial rectus combined with recession of the ipsilateral lateral rectus. Should the medial rectus not be present, then medial transposition of the vertical recti may be required (with botulinum toxin paralysis of the lateral rectus), although there is a risk of anterior segment ischaemia.

1 Mark LE, Kennerdell JS. Medial rectus injury from intranasal surgery. Arch Ophthalmol 1989;97:459-61.

2 Eitzen JP, Elsas FJ. Strabismus following endoscopic intranasal sinus surgery. $\mathcal{F}$ Pediatr Ophthalmol Strabismus 1991;28:168-70

3 Dunya IM, Salman SD, Shore JW. Ophthalmic complications of endoscopic ethmoid surgery and their management. Am f Otolaryngol 1996;17:322-31.

4 Wormald PJ, McDonagh M. The 'swing-door' technique for uncinectomy in endoscopic sinus surgery. $\mathcal{F}$ Laryngol Otol 1998;112:547-51.

5 Corey JP, Bumsted R, Panje W, et al. Orbital complications in functional endoscopic sinus surgery. Otolaryngol Head Neck Surg 1993;109:814-20.

6 May M, Levine HL, Mester SJ, et al. Complications of endoscopic sinus surgery: analysis of 2108 patients: incidence and prevention. Laryngoscope 1994;104:1080-3.

7 Lim JC, Hadfield PJ, Ghiacy S, et al. Medial orbital protrusion: a potentially hazardous anomaly during endoscopic sinus surgery. F Laryngol Otol 1999;113:754-5.

8 Buus DR, Tse DT, Farris BK. Ophthalmic complications of sinus surgery. Ophthalmology 1990;97:612-9.

9 Stankiewicz JA, Chow JM. Two faces of orbital hematoma in intranasal (endoscopic) sinu surgery. Otolaryngol Head Neck Surg 1999;120:841-7.

10 Hudgins PA. Complications of endoscopic sinus surgery. The role of the radiologist in prevention. Radiol Clin North Am 1993;31:21-32.

11 Stankiewicz JA. Blindness and intranasal endoscopic ethmoidectomy: prevention and management. Otolaryngol Head Neck Surg 1989;101:320-9. 